\title{
A Real-Time Deformable Model for Flexible Instruments Inserted into Tubular Structures
}

\author{
Markus Kukuk ${ }^{1,2}$ and Bernhard Geiger ${ }^{1}$ \\ 1 SIEMENS Corporate Research, Imaging \& Visualization, Princeton, NJ, USA \\ ${ }^{2}$ University of Dortmund, Computer science VII, Germany \\ kukuk@scr.siemens.com
}

\begin{abstract}
In this paper we present an approach to the problem of modelling long, flexible instruments, such as endoscopes or catheters. The idea is to recursively enumerate all possible shapes and subsequently filter them according to given mechanical and physical constraints. Although this brute-force approach has an exponential worst-case complexity, we show with a typical example that in case of tubular structures the empirical complexity is polynomial. We present two approximation methods that reduce this bound to a linear complexity. We have performed accuracy, runtime and robustness tests in preparation for first clinical studies.
\end{abstract}

\section{Introduction}

Flexible instruments, like endoscopes or catheters, play a central role in the field of minimally invasive surgery. They provide access to even remote operating sites within the human body through natural body openings or small incisions. However, performing endoscopic procedures or catheterizations presents a challenge to the physician. Though an endoscope has a CCD camera inside its tip that can be actively moved around by the physician, it is still difficult to derive the $3 \mathrm{D}$ position of the tip from a $2 \mathrm{D}$ video image.

In recent years numerous attempts have been made to guide endoscopic procedure by determining the position and orientation of the instrument's tip. Solomon et al. (see 1]) uses position sensors attached to the tip, Bricault et al. [2] introduced the idea of analyzing only the video images and Mori et al. 3] significantly improved this approach by achieving continuous tracking independent of the presence of strong features in the image. The authors report a processing time of 6 second per frame. Our group has recently presented a new approach to the problem [1]. We have suggested the use of a flexible endoscope model to guide a "blind" biopsy (TBNA). The model has been used in a pre-operative planning phase to derive a set of parameters that describe how to handle the endoscope in order to manoeuvre the biopsy needle inside the target. This approach requires no computer in the operating room and is inherently real-time.

A dynamic endoscope model based on multibody mechanics has been published by Ikuta [4 as part of a virtual endoscopy simulator with force sensation.

T. Dohi and R. Kikinis (Eds.): MICCAI 2002, LNCS 2489, pp. 331-338 2002.

(C) Springer-Verlag Berlin Heidelberg 2002 
Models for catheters have been published by vanWalsum et al. [5] who uses a snake-like approach and Anderson et al. 6] who uses FEM analysis.

In this paper we present a new deformable model for flexible instruments that is accurate, fast and robust. For a given insertion depth and target site, the model calculates the shape of the instrument by considering its mechanical constraints and physical environment.

\section{Model Description}

The model described in this paper consists of the following three components:

1. Discrete representation of the instrument.

2. Generator that enumerates, based on (1), all possible shapes considering given internal mechanical constraints.

3. Filter that selects from (2) only those shapes that comply with the instrument's physical constraints.

Note, that depending on the filter selectivity, more than one solution may be found. A natural way to represent a flexible tube like structure is as a chain of rigid links, interconnected by discrete ball and socket joints. A link is represented by a cylinder of certain length and diameter. A joint connects two adjacent links. If a joint restricts a link to a finite number of positions (joint positions) with respect to its predecessor, we call it a discrete joint. Link length and joint range determine the maximum flexibility of the endoscope. The mechanical constraints like varying diameter, rigid sleeves and maximum flexibility are modeled by determining for each link of the chain a suitable link diameter, length and maximum joint range.

Let $L$ be the set of all links and $\mathcal{L}=\mathcal{P}(L)$ the power set of $L$. This system can formally be described as the concatenation of two functions:

$$
I=f_{\text {filter }} \circ f_{\text {gen }}(L)
$$

with $f_{\text {gen }}: L \rightarrow \mathcal{L}$ the generator, $f_{\text {filter }}: \mathcal{L} \rightarrow \mathcal{L}$ the filter and $I \subset \mathcal{L}$ the resulting instruments. Algebraically, the generator can be described as the concatenation of two filter functions operating on $L$ :

$$
f_{\text {gen }}(L)=f_{\text {joint }}^{u, v, \theta} \circ f_{\text {link }}^{\mathbf{s}, N}(L)
$$

with $u$ the number of rotation axis and $v$ the number of discrete rotation steps of angle $\theta$ for each axis. The number of possible positions between two adjacent links is $u v$ and the joint range is $v \theta$. The parameter $\mathbf{s} \in L$ denotes the start link and $N$ the number of links (desired instrument length). Filter $f_{\text {link }}$ controls the length and size constraints and $f_{\text {joint }}$ controls the flexibility.

Function $f_{\text {filter }}$ is given as a concatenation of a geometry, tube and energy filter:

$$
f_{\text {filter }}=f_{\text {energy }}^{\alpha, \beta, p} \circ f_{\text {tube }} \circ f_{\text {geom }}
$$


with $\alpha, \beta$ two material constants for bending and torsion and $p$ the filter selectivity. Filter $f_{\text {geom }}$ filters those links that collide with the organ wall and $f_{\text {tube }}$ represents a simple bounding tube filter, which based on an insertion protocol defines a ROI in case of the existence of bifurcations. Finally, filter $f_{\text {energy }}$ finds the global minima (for $p=1$ ) regarding the instrument's deformation energy:

$$
f_{\text {energy }}=\left\{A \in \mathcal{L} \mid\left(E_{\kappa}(A)+E_{\tau}(A)\right)=\min _{p}\right\}
$$

with $\min _{p}$ reading "among the $p$ smallest values", $E_{\kappa}$ the internal bending energy and $E_{\tau}$ the internal torsion energy of an instrument. The two discrete energy terms are given by:

$$
E_{\kappa}(A)=\sum_{i=1}^{N-1} \alpha \kappa(A, i)^{2} \quad \text { and } \quad E_{\tau}(A)=\sum_{i=1}^{N-1} \beta \tau(A, i)^{2}
$$

with $\alpha$ the amount of resistance to bending, $\kappa(A, i)$ the angle between link $i$ and $i+1$ of an instrument $A, \beta$ the amount of resistance to twisting and $\tau(A, i)$ the torsion between link $i$ and $i+1$ of an instrument $A$.

The physical basis for this model is that we regard an endoscope as an elastic structure, which obeys Hooke's law. Although our model reflects quite well the instrument's mechanical structure, it disregards external factors like organ deformation, friction etc. Instead, we accept some degree of fuzziness regarding the exact tip location and try to cover this fuzziness by computing a set of possible tip shapes. A natural way to expand the solution set of our model, is to relax the selectivity of filter $f_{\text {energy }}$ in the sense that it determines the shapes of the $p>1$ smallest energies, rather than just one shape of minimum energy.

\subsection{Implementation}

We introduced the concept of modelling a flexible instrument by enumerating all possible shapes and filtering the result according to given constraints. A natural way to implement this concept is to recursively create a spatial tree (depthfirst search backtracking), whose growth is constraint according to a set of filter functions. A spatial tree is an ordinary tree data structure, where each node represents a joint in 3D space. Each edge that connects a node with its child represents a link in 3D space. Each path from the root to a leaf represents a chain of links and therewith a flexible instrument. The entire spatial tree, so all path from the root to the leafs represent the instrument's full workspace under the given constraints.

A link is represented by a coordinate system attached to a cylinder with length $c$ and diameter $d$. The coordinate system is attached to the cylinder, in a way that it's $z$-axis is the centerline of the cylinder. The cylinder's bottom and top bases lie in the $z=0$ and $z=c$ plane.

An algorithm $f_{\text {gen }}$ that creates such a spatial tree, takes a link $\mathbf{l} \in L$ as input, attaches $u v$ links to $\mathbf{l}$ and recursively calls $f_{\text {gen }}$ for each attached link:

$$
f_{\text {gen }}(\mathbf{l})=f_{\text {gen }}\left(\mathrm{R}_{i, j} \mathbf{l} \mathrm{T}(c)\right) \text { for } i=1, \ldots, u, j=1, \ldots, v
$$



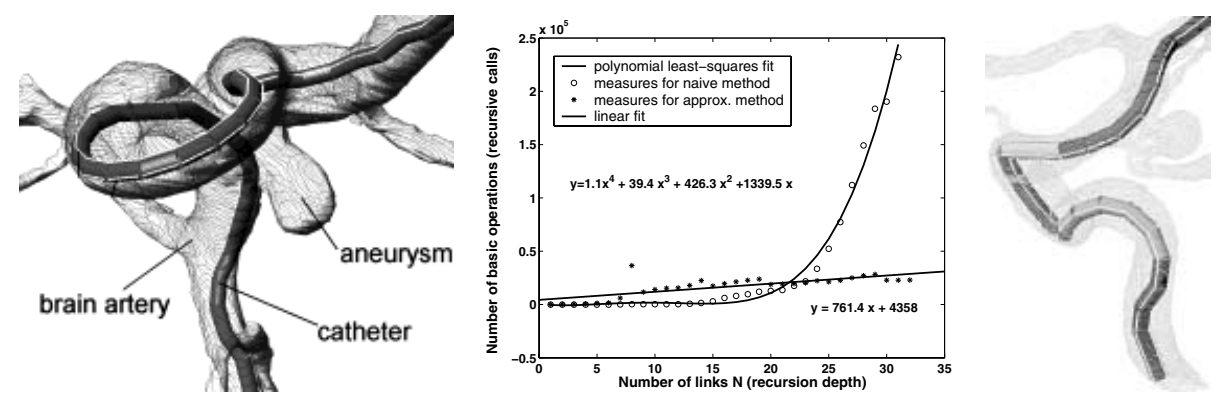

Fig. 1. Measured complexity of a catheter inserted into a brain artery. Left: Naive method. Right: Same data set using $(n, k)$ - and $(u, v)$ - approximation. Center: Fourthorder polynomial model for the naive method. Linear model for the approximation.

with $\mathbf{T}(c)$ a translation matrix that moves $\mathbf{l}$ a distance of $c \mathrm{~mm}$ along its main axis towards the link end and $\mathrm{R}_{i, j}$ a rotation matrix that rotates $\mathbf{l}$ into the new position. The rotation matrix is given as an entry in a pre-computed look-up table of $u$ columns and $v$ rows:

$$
\mathrm{R}_{i, j}=\mathrm{R}\left(\boldsymbol{r}_{i}, j \theta\right) \text { for } i=1, \ldots, u, j=1, \ldots, v
$$

with $j \theta$ the rotation angle and $\boldsymbol{r}_{i}$ the $i$-th rotation axis. For example for $u=9$ :

$$
\boldsymbol{r}_{i} \in\left\{\left(\begin{array}{lll}
x & y & 0
\end{array}\right)^{T} \mid x, y \in\{-1,0,1\}\right\}
$$

\subsection{Complexity}

We propose to create a spatial tree to enumerate all shapes an endoscope can take, given a start link and a maximum number of $N$ links. The time and space complexity for this naive approach is $O\left((u v)^{N}\right)$.

However, depending on how much the growth of the spatial tree is constraint by the filter functions, the practical complexity, given a real anatomy, is more feasible. Especially tubular structures such as the tracheobronchial tree and the vasculature greatly limit the growth of the tree. The following experiment confirms this hypothesis: For a fix start position, we use our flexible instrument model $(u=9, v=1)$ to calculate a catheter of length $\mathrm{N}$, inserted into a brain artery. We do this 31 times, for $N=1, \ldots, 31$. For each calculation we count the number of recursive calls needed to enumerate all possibilities, given the geometry and energy filter. As shown in Fig. 1 a fourth-order polynomial can be fit (least squares) to the resulting curve, indicating that the real complexity for this anatomy is $O\left(N^{4}\right)$. For this naive method, 17 links could be computed in less than one second on a Pentium 4, $1.3 \mathrm{GHz}$ processor. The second pair of curves shows the complexity after activation of two approximation methods described in the following two sections. 


\section{$2.3 \quad(n, k)$-Approximation}

We have shown with a typical example that the actual complexity appears to be polynomial $\left(O\left(N^{4}\right)\right)$ for tubular structures, instead of exponential. We have also shown that for a considerable number of links $n<<N$ computation can be done in real-time, given off-the-shelf PC hardware. A straight forward idea for accelerating the computation, is to compose an instrument of length $N$ from several instrument segments of length $n$.

The idea is, to calculate an instrument of length $N$, by connecting together $\left\lceil\frac{N}{k}\right\rceil$ segments of length $k$. Each segment of length $k$ is obtained by simply taking the first $k$ links of an "sub-instrument" of length $n$. The start link of each sub-instrument is given by the $k$-th link of the previous sub-instrument. All root-to-leaf paths of a sub-instrument's spatial tree can be regarded as a set of "tentacles", that reach out to explore the environment ahead. Formally:

$$
I(\mathbf{s}, N)=\bigcup_{i=1}^{\left\lceil\frac{N}{k}\right\rceil} I_{i}\left(\mathbf{s}_{\mathbf{i}}, n\right)[0, \ldots, k-1] \text { with } \mathbf{s}_{\mathbf{0}}=\mathbf{s}, \quad \mathbf{s}_{\mathbf{i}}=I_{i-1}\left(\mathbf{s}_{\mathbf{i}-\mathbf{1}}, n\right)[k]
$$

$I(\mathbf{s}, N)$ is the resulting instrument with start link $\mathbf{s}$ and length $N$ and $I_{i}()$ the $i$-th "sub-instrument". The notation $I()[i]$, resp. $I()[i, \ldots, j]$ denotes the i-th, resp. i-th to j-th link (head to tip) of instrument $I$. The new complexity is:

$$
O\left(\left\lceil\frac{N}{k}\right\rceil(u v)^{n}\right) \leq O\left((u v)^{N}\right)
$$

Fig. 2 top right shows an example with $n=16$ and $k=2$. It shows as an intermediate result the first 13 segments, which means that 26 links out of $N=$ 44 have been computed. For the bottom figure $n=20$ and $k=4$. To take account of the frictional forces acting on the endoscope's tip, we set $p=7$ (eqn. (4) for the last segment and $p=1$ for all others.

\section{$2.4(u, v)$-Approximation}

We now describe a technique to further speed up the computation. It is based on the observation that the energy filter favors configurations with many small joint angles instead of a few big angles. In other words it tends to distribute bending on many joints, using a small angle for each joint. As a consequence the dispersion of all angles tends to be small. This is particularly true for short tentacles of length $n<<N$ used in the $(n, k)$-approximation scheme. The idea now is, to constrain for each execution of the model the maneuverability of each joint to only one possible angle and to stepwise increase this angle between subsequent executions:

$$
I_{h}(\mathbf{s}, n, j) \text { for } j=1, \ldots, v \text { with } f_{\text {gen }}(\mathbf{l}, j)=f_{\text {gen }}\left(\mathrm{R}_{i, j} \mathbf{l} \mathrm{T}(c)\right) \text { for } i=1, \ldots, u
$$

with $I_{h}()$ the "sub-instrument" of equation 9 extended by a parameter $j$. Note, that a $0^{\circ}$ angle is included in the $u$ principal directions. The resulting configuration is the one with minimum energy among the $v$ executions of $I_{h}()$. The new complexity is given by: 


$$
O\left(\left\lceil\frac{N}{k}\right\rceil u^{n} v\right) \leq O\left(\left\lceil\frac{N}{k}\right\rceil(u v)^{n}\right)
$$

Fig. 1]shows a linear complexity for $N=32, n=10, k=5, u=9, v=20, \theta=2^{\circ}$.

\section{$3 \quad$ Experiments}

\subsection{Model Calibration and Validation}

Objective: We describe an experiment to determine the intrinsic model parameters. The idea is, to measure the center line of a real endoscope inserted into a calibration phantom and to generate a matching virtual endoscope by finding suitable values for the model parameters.

Material: Hardware, see Fig. 2, left: Optical tracking system ARTtrack 1 (A.R.T. GmbH, www.ar-tracking.de), comprising two IR cameras and a set of passive, retro-reflective markers. Video endoscope OLYMPUS GIF-100 (9.5 mm diameter) with 30 stripe markers (10 mm width) wrapped around it's shaft like a ring in a distance of ca. $25 \mathrm{~mm}$. Board with a "M"-shaped calibration path, $1050 \mathrm{~mm}$ long, $50 \mathrm{~mm}$ wide. We have attached three disk markers to the board. Pointing device with a calibrated tip.

Design and Methods: (1) Since the tracking system is designed to determine the center of either ball or disk markers, we have to verify it's accuracy in measuring the center of our ring markers. The idea is to verify whether the center line of the endoscope (given by the center of each ring marker) is in a distance to the board according to the radius of the endoscope. The board surface plane is determined using the three disk markers.

(2) We insert the endoscope a distance of $900 \mathrm{~mm}$ into the "M" path. After the insertion, we record a reading of all endoscope markers. To draw the measured markers and the digital model of the " $\mathrm{M}$ " in one common reference frame, we need to find a rigid body transformation that maps one frame into the other. To find the transform, we calculate the best fit in the least-squares sense between a set of reference points obtained with the pointer and the corresponding virtual points. To solve the resulting non-linear minimization problem, we use the "Levenberg-Marquardt" method.

Results: (1) We are able to accurately determine the center line of a real endoscope using ring markers. The tracking system could determine the position of 26 out of 30 markers. The average distance between the markers and the board is $5.17 \mathrm{~mm}$ (SD: $0.8 \mathrm{~mm}$ ), given a $4.75 \mathrm{~mm}$ shaft radius and a $0.5 \mathrm{~mm}$ marker thickness. (2) Fig. 2, bottom right shows the best match between the markers (black balls) and the model. The model consists of 9 segments (color coded), the first 8 consist of 4 , the last consists of 12 links. Each link is $20.45 \mathrm{~mm}$ long. By calculating the 7 smallest energies for the last segment, we obtained one segment that matched the last 6 markers by a Hausdorff distance of $0.8 \mathrm{~mm}$. 

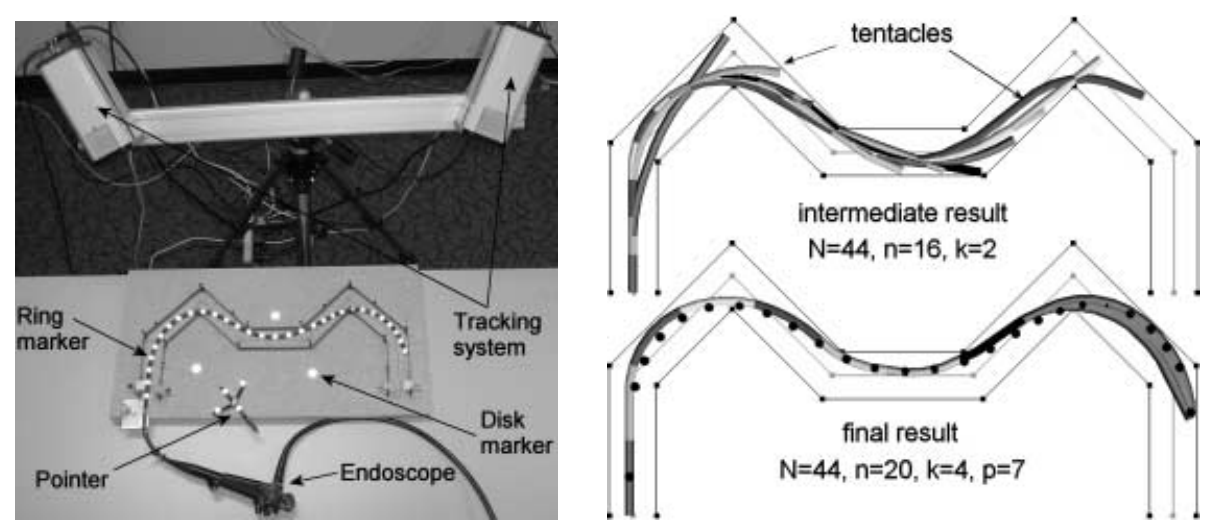

Fig. 2. Left: Experimental setup (photo, flash). Right: Screen shots. Top: Resulting tentacles $(p=1)$ of the first 13 segments. Bottom: Real endoscope (black balls) and final result showing the 7 smallest energies for the last segment.

\subsection{Accuracy, Run-time and Robustness Tests}

Material: In addition to the hardware from the previous experiment: Phantom of the tracheobronchial tree made of transparent plastic tubes. We placed 37 sticks as reference points for the rigid body transformation in the model.

Software: The phantom was scanned (CT, 512x512x382, $1 \mathrm{~mm}$ slice distance, $1.2 \mathrm{~mm}$ thickness), the reference points were manually identified and a lung phantom model was reconstructed (ca. 13000 triangles).

Design and Methods: (1) To asses the influence of refraction caused by the plastic tubes on the measures, we have inserted a calibration wand with markers of known distance into the phantom. (2) We insert the endoscope into different branches of the phantom (see Fig. 3 (a)) and record the position of the markers for different insertion depths. The position and orientation of the real endoscope's tip is given by it's first rigid sleeve (d), to which we have attached two markers. To asses the accuracy of our model, we compare these two markers to the position and orientation of the corresponding virtual sleeve. We have performed 5 tests and for each we have calculated the best result regarding a match between 10 virtual sleeves $(p=10)$ and the real sleeve.

Results: (1) The influence of refraction is within the accuracy of the tracking system $(0.1 \mathrm{~mm})$. (2) The worst result out of the 5 best results is $2.5 \mathrm{~mm}$ (position) and $7^{\circ}$ (orientation). The best result is $0.7 \mathrm{~mm}$ and $4^{\circ}$. The time needed to calculate and display the model shown in Fig. 3 (b) with a maximum insertion depth of $355 \mathrm{~mm}$ is 0.6 seconds on a Pentium $4,1.3 \mathrm{GHz}$. We were able to generate a continuous animation of a $240 \mathrm{~mm}$ long insertion, by increasing the insertion depth by $1 \mathrm{~mm}$, which demonstrates the algorithm's robustness. Computation and display time for the animation (240 frames) is 27 seconds. 

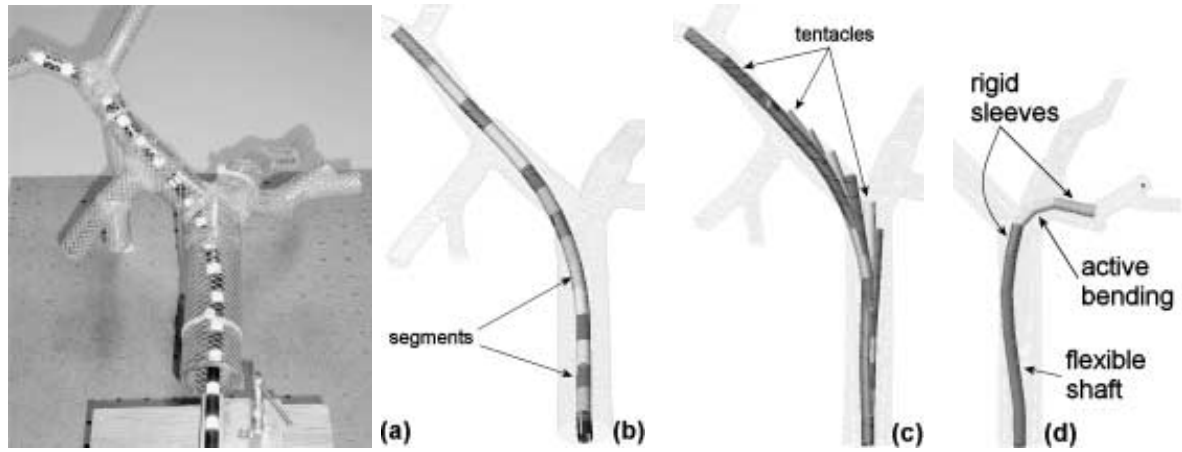

Fig. 3. (a) OLYMPUS GIF-100 endoscope with reflective markers inserted into a lung phantom. (b) Model of the GIF-100 inside the same branch as in (a). (c) Tentacles shown for each segment. (d) The two rigid sleeves (35 and $25 \mathrm{~mm}$ long) of the GIF-100 digital model. The model's tip was bent by $90^{\circ}$ to reach into the smaller branch.

\section{Conclusion}

We have presented a deformable model for flexible instruments. The model reflects special mechanical constraints often found with flexible instruments, like a tip that can be bent to a much higher degree than the shaft (Fig. 3 (d)), rigid sleeves within flexible sections and a non-negligible shaft diameter. We demonstrated its use as a catheter inserted into vasculature and as an endoscope inserted into the tracheobronchial tree. An interesting property of our model is the option to generate several possible shapes for the instrument's tip. Furthermore, it requires no initialization in form of an initial "good guess" (like snake approach) for the final shape and no preprocessing (like FEM approach).

\section{References}

1. Markus Kukuk et al. TBNA-protocols - Guiding TransBronchial Needle Aspirations Without a Computer in the Operating Room. In MICCAI '01 - LNCS 2208, pages 997-1006. Springer.

2. I. Bricault, G. Ferretti, and P. Cinquin. Multi-level Strategy for Computer-Assisted Transbronchial Biopsy. In MICCAI, volume 1496 of LNCS. Springer, 1998.

3. Kensaku Mori et al. A Method for Tracking the Camera Motion of Real Endoscope by Epipolar Geometry Analysis and Virtual Endoscopy System. In MICCAI, volume 2208 of $L N C S$, pages 1-8. Springer, 2001.

4. Koji Ikuta et al. Portable Virtual Endoscope System with Force and Visual Display for Insertion Training. In MICCAI, volume 1935 of LNCS. Springer, 2000.

5. Theo van Walsum et al. Deformable B-splines for catheter simulation. In CARS'99, 1999.

6. J. Anderson, W. Brody, et al. daVinci - A vascular catheterization and interventional radiology-based training and patient pretreatment planning simulator. In Proc. of Society of Cardiovascular and Interventional Radiology (SCVIR), March 1996. 